\title{
Linear-time computation of minimal absent words using suffix array
}

\author{
Carl Barton ${ }^{1}$, Alice Heliou ${ }^{2,3}$, Laurent Mouchard ${ }^{4}$ and Solon P Pissis ${ }^{1 *}$
}

\begin{abstract}
Background: An absent word of a word $y$ of length $n$ is a word that does not occur in $y$. It is a minimal absent word if all its proper factors occur in $y$. Minimal absent words have been computed in genomes of organisms from all domains of life; their computation also provides a fast alternative for measuring approximation in sequence comparison. There exists an $\mathcal{O}(n)$-time and $\mathcal{O}(n)$-space algorithm for computing all minimal absent words on a fixed-sized alphabet based on the construction of suffix automata (Crochemore et al., 1998). No implementation of this algorithm is publicly available. There also exists an $\mathcal{O}\left(n^{2}\right)$-time and $\mathcal{O}(n)$-space algorithm for the same problem based on the construction of suffix arrays (Pinho et al., 2009). An implementation of this algorithm was also provided by the authors and is currently the fastest available.
\end{abstract}

Results: Our contribution in this article is twofold: first, we bridge this unpleasant gap by presenting an $\mathcal{O}(n)$-time and $\mathcal{O}(n)$-space algorithm for computing all minimal absent words based on the construction of suffix arrays; and second, we provide the respective implementation of this algorithm. Experimental results, using real and synthetic data, show that this implementation outperforms the one by Pinho et al. The open-source code of our implementation is freely available at http://github.com/solonas 13/maw.

Conclusions: Classical notions for sequence comparison are increasingly being replaced by other similarity measures that refer to the composition of sequences in terms of their constituent patterns. One such measure is the minimal absent words. In this article, we present a new linear-time and linear-space algorithm for the computation of minimal absent words based on the suffix array.

Keywords: Absent words, Minimal absent words, Suffix array

\section{Background}

Sequence comparison is an important step in many important tasks in bioinformatics. It is used in many applications; from phylogenies reconstruction to the reconstruction of genomes. Traditional techniques for measuring approximation in sequence comparison are based on the notions of distance or of similarity between sequences; and these are computed through sequence alignment techniques. An issue with using alignment techniques is that they are computationally expensive: they require quadratic time in the length of the sequences. Moreover, in molecular taxonomy and phylogeny, for instance, whole-genome alignment proves both

\footnotetext{
${ }^{*}$ Correspondence: solon.pissis@kcl.ac.uk

1 Department of Informatics, King's College London, The Strand, WC2R 2LS London, UK

Full list of author information is available at the end of the article
}

computationally expensive and hardly significant. These observations have led to increased research into alignment free techniques for sequence comparison. A number of alignment free techniques have been proposed: in [1], a method based on the computation of the shortest unique factors of each sequence is proposed; other approaches estimate the number of mismatches per site based on the length of exact matches between pairs of sequences [2].

Thus standard notions are gradually being complemented (or even supplanted) by other measures that refer, implicitly or explicitly, to the composition of sequences in terms of their constituent patterns. One such measure is the notion of words absent in a sequence. A word is an absent word of some sequence if it does not occur in the sequence. These words represent a type of negative information: information about what does not occur in the sequence. Noting the words which do occur in one 
sequence but do not occur in another can be used to detect mutations or other biologically significant events.

Given a sequence of length $n$, the number of absent words of length at most $n$ can be exponential in $n$, meaning that using all the absent words for sequence comparison is more expensive than alignments. However, the number of certain subsets of absent words is only linear in $n$. An absent word of a sequence is a shortest absent word if all words shorter than it do occur in the sequence. An $\mathcal{O}(m n)$-time algorithm for computing shortest absent words was presented in [3], where $m$ is a user-specified threshold on the length of the shortest absent words. This was later improved by [4], who presented an $\mathcal{O}(n \log \log n)$-time algorithm for the same problem. This has been further improved and an $\mathcal{O}(n)-$ time algorithm was presented in [5].

A minimal absent word of a sequence is an absent word whose proper factors all occur in the sequence. Notice that minimal absent words are a superset of shortest absent words [6]. An upper bound on the number of minimal absent words is $\mathcal{O}(\sigma n)[7,8]$, where $\sigma$ is the size of the alphabet. This suggests that it may be possible to compare sequences in time proportional to their lengths, for a fixed-sized alphabet, instead of proportional to the product of their lengths [1]. Theory and some applications of minimal absent words can be found in [9].

Recently, there has been a number of biological studies on the significance of absent words. The most comprehensive study on the significance of absent words is probably [10]; in this, the authors suggest that the deficit of certain subsets of absent words in vertebrates may be explained by the hypermutability of the genome. It was later found in [11] that the compositional biases observed in [10] for vertebrates are not uniform throughout different sets of minimal absent words. Moreover, the analyses in [11] support the hypothesis of the inheritance of minimal absent words through a common ancestor, in addition to lineage-specific inheritance, only in vertebrates. In [12], the minimal absent words in four human genomes were computed, and it was shown that, as expected, intra-species variations in minimal absent words were lower than inter-species variations. Minimal absent words have also been used for phylogenies reconstruction [13].

From an algorithmic perspective, an $\mathcal{O}(n)$-time and $\mathcal{O}(n)$-space algorithm for computing all minimal absent words on a fixed-sized alphabet based on the construction of suffix automata was presented in [7]. An alternative $\mathcal{O}(n)$-time solution for finding minimal absent words of length at most $\ell$, such that $\ell=\mathcal{O}(1)$, based on the construction of tries of bounded-length factors was presented in [13]. A drawback of these approaches, in practical terms, is that the construction of suffix automata (or of tries) may have a large memory footprint. Due to this, an important problem is to be able to compute the minimal absent words of a sequence without the use of data structures such as the suffix automaton. To this end, the computation of minimal absent words based on the construction of suffix arrays was considered in [6]; although fast in practice, the worstcase runtime of this algorithm is $\mathcal{O}\left(n^{2}\right)$. Alternatively, one could make use of the succinct representations of the bidirectional BWT, recently presented in [14], to compute all minimal absent words in time $\mathcal{O}(n)$. However, an implementation of these representations was not made available by the authors; and it is also rather unlikely that such an implementation will outperform an $\mathcal{O}(n)$-time algorithm based on the construction of suffix arrays.

\section{Our contribution}

In this article, we bridge this unpleasant gap by presenting the first $\mathcal{O}(n)$-time and $\mathcal{O}(n)$-space algorithm for computing all minimal absent words of a sequence of length $n$ based on the construction of suffix arrays. In addition, we provide the respective implementation of this algorithm. This implementation is shown to be more efficient than existing tools, both in terms of speed and memory.

\section{Methods}

\section{Definitions and notation}

To provide an overview of our result and algorithm, we begin with a few definitions. Let $y=y[0] y[1] . . y[n-1]$ be a word of length $n=|y|$ over a finite ordered alphabet $\Sigma$ of size $\sigma=|\Sigma|=\mathcal{O}(1)$. We denote by $y[i . j]=y[i] . . y[j]$ the factor of $y$ that starts at position $i$ and ends at position $j$ and by $\varepsilon$ the empty word, word of length 0 . We recall that a prefix of $y$ is a factor that starts at position $0(y[0 . j])$ and a suffix is a factor that ends at position $n-1(y[i . . n-1])$, and that a factor of $y$ is a proper factor if it is not the empty word or $y$ itself.

Let $x$ be a word of length $0<m \leq n$. We say that there exists an occurrence of $x$ in $y$, or, more simply, that $x$ occurs in $y$, when $x$ is a factor of $y$. Every occurrence of $x$ can be characterised by a starting position in $y$. Thus we say that $x$ occurs at the starting position $i$ in $y$ when $x=y[i . i+m-1]$. Opposingly, we say that the word $x$ is an absent word of $y$ if it does not occur in $y$. The absent word $x, m \geq 2$, of $y$ is minimal if and only if all its proper factors occur in $y$.

We denote by SA the suffix array of $y$, that is the array of length $n$ of the starting positions of all sorted suffixes of $y$, i.e. for all $1 \leq r<n$, we have $y[\mathrm{SA}[r-1] . . n-1]<$ $y[\mathrm{SA}[r] . . n-1][15]$. Let $\operatorname{Icp}(r, s)$ denote the length of the longest common prefix of the words $y[\mathrm{SA}[r] . . n-1]$ and $y[\mathrm{SA}[s] . . n-1]$, for all $0 \leq r, s<n$, and 0 otherwise. We 


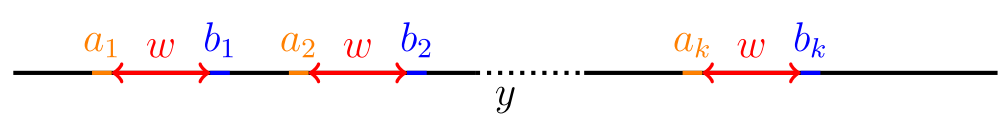

Figure $1 \boldsymbol{k}$ occurrences of a factor $\boldsymbol{w}$ of $\boldsymbol{y}$; they are preceded by $\boldsymbol{a}_{\boldsymbol{i}}$ and followed by $\boldsymbol{b}_{\boldsymbol{i}}$. If there exist $i, j \in[1: k]$ such that $\left(a_{i}, b_{j}\right) \notin\left\{\left(a_{1}, b_{1}\right), \ldots\right.$, $\left.\left(a_{k}, b_{k}\right)\right\}$ then $a_{i} w b_{j}$ is a minimal absent word of $y$.

denote by LCP the longest common prefix array of $y$ defined by $L C P[r]=\operatorname{Icp}(r-1, r)$, for all $1 \leq r<n$, and $L C P[0]=0$. The inverse iSA of the array SA is defined by iSA $[\mathrm{SA}[r]]=$ $r$, for all $0 \leq r<n$. SA [16], iSA, and LCP [17] of $y$ can be computed in time and space $\mathcal{O}(n)$.

In this article, we consider the following problem:

\section{MinimALABSENTWORDS}

Input: a word $y$ on $\Sigma$ of length $n$

Output: for every minimal absent word $x$ of $y$, one tuple $<a,(i, j)\rangle$, such that $x$ is defined by $x[0]=a, a \in \Sigma$, and $x[1 . . m-1]=y[i . . j], m \geq 2$.

\section{Algorithm MAW}

In this section, we present algorithm MAW, an $\mathcal{O}(n)$-time and $\mathcal{O}(n)$-space algorithm for finding all minimal absent words in a word of length $n$ using arrays SA and LCP.

We first give an example and explain how we can characterise the minimal absent words; then we introduce how their computation can be done efficiently by using arrays SA and LCP. Finally, we present in detail the two main steps of the algorithm.

Intuitively, the idea is to look at the occurrences of a factor $w$ of $y$ and, in particular, at the letters that precede and follow these occurrences. If we find a couple $(a, b)$, $a, b \in \Sigma$, such that $a w$ and $w b$ occur in $y$, but $a w b$ does not occur in $y$, then we can conclude that $a w b$ is a minimal absent word of $y$. For an illustration inspect Figure 1.

For example, let us consider the word $y=\mathrm{AABABABB}$ :

- factor $w=\mathrm{AB}$ occurs at:

- position 1 preceded by $\mathrm{A}$ and followed by $\mathrm{A}$

- position 3 preceded by $B$ and followed by $A$

- position 5 preceded by B and followed by B

We see that $\mathrm{A} w$ occurs and $w \mathrm{~B}$ occurs as well but $\mathrm{A} w \mathrm{~B}$ does not occur in $y$, so $\mathrm{AABB}$ is a minimal absent word of $y$.

- factor $w=$ BA occurs at:

- position 2 preceded by $\mathrm{A}$ and followed by $\mathrm{B}$

- position 4 preceded by $A$ and followed by $B$

We cannot infer a minimal absent word.

A minimal absent word $x[0 . . m-1]$ of a word $y[0 . . n-1]$ is an absent word whose proper factors all occur in $y$. Among them, $x_{1}=x[1 . . m-1]$ and $x_{2}=x[1 . . m-2]=$ $x_{1}\left[0 . .\left|x_{1}\right|-2\right]$ occur in $y$ (inspect Figure 2); we will focus on these two factors to characterise the minimal absent words. To do so, we will consider each occurrence of $x_{1}$ and $x_{2}$, and construct the sets of letters that occur just before:

$\mathrm{B}_{1}\left(x_{1}\right)=\{y[j-1]: j$ is the starting position of an occurrence of $\left.x_{1}\right\}$

$\mathrm{B}_{2}\left(x_{1}\right)=\{y[j-1]: j$ is the starting position of an occurrence of $\left.x_{1}\left[0 . .\left|x_{1}\right|-2\right]\right\}$.

Lemma 1. Let $x$ and $y$ be two words. Then $x$ is a minimal absent word of $y$ if and only if $x[0]$ is an element of $B_{2}\left(x_{1}\right)$ and not of $B_{1}\left(x_{1}\right)$, with $x_{1}=x[1 . . m-1]$.

Proof. $(\Rightarrow)$ Let $x_{1}$ be a factor of $y, x_{2}$ be the longest proper prefix of $x_{1}$, and $B_{1}\left(x_{1}\right)$ and $\mathrm{B}_{2}\left(x_{1}\right)$ the sets defined above. Further let $p$ be a letter that is in $B_{2}\left(x_{1}\right)$ but not in $\mathrm{B}_{1}\left(x_{1}\right)$. Then, there exists a starting position $j$ of an occurrence of $x_{2}$ such that $y[j-1]=p$, so the word $p x_{2}$ occurs at position $j-1$ in $y \cdot p$ is not in $\mathrm{B}_{1}\left(x_{1}\right)$ so $p x_{1}$ does not occur in $x$ and is therefore an absent word of $y . x_{1}$ and $p x_{2}$ are factors of $y$, so all the proper factors of $p x_{1}$ occur in $y$, thus $p x_{1}$ is a minimal absent word of $y$.

$(\Leftarrow)$ Let $x[0 . . m-1]$ be a minimal absent word of $y$. Its longest proper prefix $x[0 . . m-2]=x[0] x_{1}\left[0 . .\left|x_{1}\right|-2\right]$ occurs in $y$, so $x[0]$ is in $\mathrm{B}_{2}\left(x_{1}\right)$. Its longest proper suffix, $x_{1}$ occurs as well in $y$, but $x=x[0] x_{1}$ is an absent word of $y$ so it does not occur in $y$ and $x[0]$ is not in $B_{1}\left(x_{1}\right)$.

Lemma 2. Let $x$ be a minimal absent word of length $m$ of word $y$ of length $n$. Then there exists an integer $i \in$ $[0: n-1]$ such that $y[\mathrm{SA}[i] . \mathrm{SA}[i]+\mathrm{LCP}[i]]=x_{1}$ or $y[\mathrm{SA}[i] . . \mathrm{SA}[i]+\mathrm{LCP}[i+1]]=x_{1}$, where $x_{1}=x[1 . . m-1]$.

Proof. Let $j$ be the starting position of an occurrence of $x[0 . m-2]$ in $y$ and $k$ the starting position of an

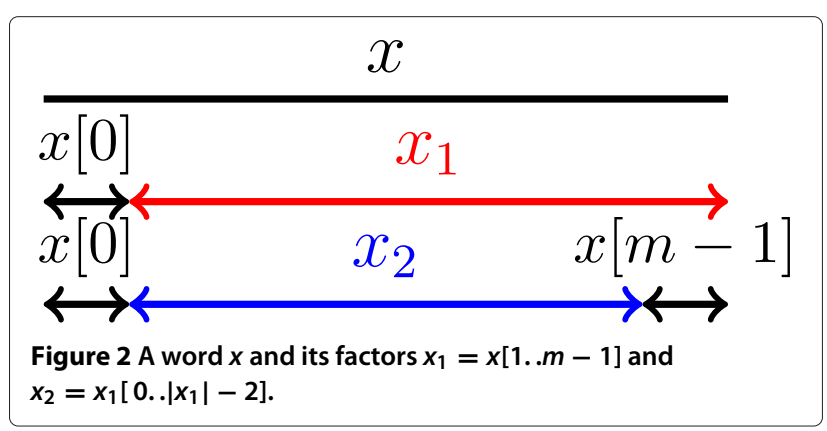


occurrence of $x_{1}$ in $y$. The suffixes $y[j+1 . . n-1]$ and $y[k . . n-1]$ share $x_{2}=x[1 . . m-2]$ as a common prefix. As $x$ is an absent word of $y$, this common prefix cannot be extended so $x_{2}$ is the longest common prefix of those suffixes. By using iSA, the inverse suffix array, we have $\operatorname{Icp}(\mathrm{iSA}[j+1], \mathrm{iSA}[k])=m-2$. Let us also note $s_{k}=\mathrm{iSA}[k]$ and $s_{j+1}=\mathrm{iSA}[j+1]$. We then have two possibilities:

- if $s_{k}>s_{j+1}$ : for all $s$ in $\left[s_{j+1}+1: s_{k}\right]$, we have $\mathrm{LCP}[s] \geq m-2$, with equality holding for at least one position. Let us define $i=\max \left\{s \in\left[s_{j+1}+1: s_{k}\right]\right.$ : $\mathrm{LCP}[s]=m-2\}$, the maximality of $i$ implies that $i=s_{k}$ or $\operatorname{Icp}\left(i, s_{k}\right)>m-2$ and thus, in both cases $y[\mathrm{SA}[i] . . \mathrm{SA}[i]+\mathrm{LCP}[i]]=x_{1}$.

- if $s_{j+1}>s_{k}$ : for all $s$ in $\left[s_{k}+1: s_{j+1}\right]$, we have $\mathrm{LCP}[s] \geq m-2$, with equality holding for at least one position. Let us define $i=\min \left\{s \in\left[s_{k}: s_{j+1}-1\right]\right.$ : $\mathrm{LCP}[s+1]=m-2\}$, the minimality of $i$ implies $i=s_{k}$ or $\operatorname{lcp}\left(s_{k}, i\right)>m-2$ and thus, in both cases $y[\mathrm{SA}[i] . . \mathrm{SA}[i]+\mathrm{LCP}[i+1]]=x_{1}$.

For an illustration inspect Figure 3.

By Lemma 2, we can compute all minimal absent words of $y$ by examining only the factors $S_{2 i}=$ $y[\mathrm{SA}[i] . . \mathrm{SA}[i]+\mathrm{LCP}[i]]$ and $S_{2 i+1}=y[\mathrm{SA}[i] . . \mathrm{SA}[i]+$ $\mathrm{LCP}[i+1]]$, for all $i$ in $[0: n-1]$. We just need to construct the sets $\mathrm{B}_{1}\left(S_{2 i}\right), \mathrm{B}_{2}\left(S_{2 i}\right)$ and $\mathrm{B}_{1}\left(S_{2 i+1}\right), \mathrm{B}_{2}\left(S_{2 i+1}\right)$, where $\mathrm{B}_{1}\left(S_{j}\right)$ (resp. $\mathrm{B}_{2}\left(S_{j}\right)$ ) is the set of letters that immediately precede an occurrence of the factor $S_{j}$ (resp. the longest proper prefix of $S_{j}$ ), for all $j$ in $[0: 2 n-1]$. Then, by Lemma 1 , the difference between $\mathrm{B}_{2}\left(S_{j}\right)$ and $\mathrm{B}_{1}\left(S_{j}\right)$, for all $j$ in $[0: 2 n-1]$, gives us all the minimal absent words of $y$.

Thus the important computational step is to compute these sets of letters efficiently. To do so, we visit twice arrays $\mathrm{SA}$ and LCP using another array denoted by $\mathrm{B}_{1}$ (resp. $\mathrm{B}_{2}$ ) to store set $\mathrm{B}_{1}\left(S_{j}\right)$ (resp. $\mathrm{B}_{2}\left(S_{j}\right)$ ), for all $j$ in $[0: 2 n-1]$. Both arrays $B_{1}$ and $B_{2}$ consist of $2 n$ elements, where each element is a bit vector of length $\sigma$, the size of the alphabet, corresponding to one bit per alphabet letter. While iterating over arrays SA and LCP, we maintain another array denoted by Interval, such that, at the end of each iteration $i$, the $\ell^{\text {th }}$ element of Interval stores the set of letters we have encountered before the prefix of length $\ell$ of $y[\mathrm{SA}[i] . . n-1]$. Array Interval consists of $\max _{i \in[0: n-1]} \mathrm{LCP}[i]+1$ elements, where each element is a bit vector of length $\sigma$.

During the first pass, we visit arrays SA and LCP from top to bottom. For each $i \in[0: n-1]$, we store in positions $2 i$ and $2 i+1$ of $\mathrm{B}_{1}$ (resp. $\mathrm{B}_{2}$ ) the set of letters that immediately precede occurrences of $S_{2 i}$ and $S_{2 i+1}$ (resp. their longest proper prefixes) whose starting positions appear before position $i$ in SA. During the second pass, we go bottom up to complete the sets, which are already stored, with the letters preceding the occurrences whose starting positions appear after position $i$ in SA. In order to be efficient, we will maintain a stack structure, denoted by LifoLCP, to store the LCP values of the factors that are prefixes of the one we are currently visiting.

\section{Top-down pass}

Each iteration of the top-down pass consists of two steps. In the first step, we visit LifoLCP from the top and for each $L C P$ value read we set to zero the corresponding element of Interval; then we remove this value from the stack. We stop when we reach a value smaller or equal to LCP $[i]$. We do this as the corresponding factors are not prefixes of $y[\mathrm{SA}[i] . . n-1]$, nor will they be prefixes in the remaining suffixes. We push at most one value onto the stack LifoLCP per iteration, so, in total, there are $n$ times we will set an element of Interval to zero. This step requires time and space $\mathcal{O}(n \sigma)$.

For the second step, we update the elements that correspond to factors in the suffix array with an LCP value less than $L C P[i]$. To do so, we visit the stack LifoLCP top-down and, for each LCP value read, we add the letter $y[S A[i]-1]$ to the corresponding element of Interval until we reach a value whose element already contains it. This ensures that, for each value read, the corresponding element of Interval has no more than $\sigma$ letters added. As we consider at most
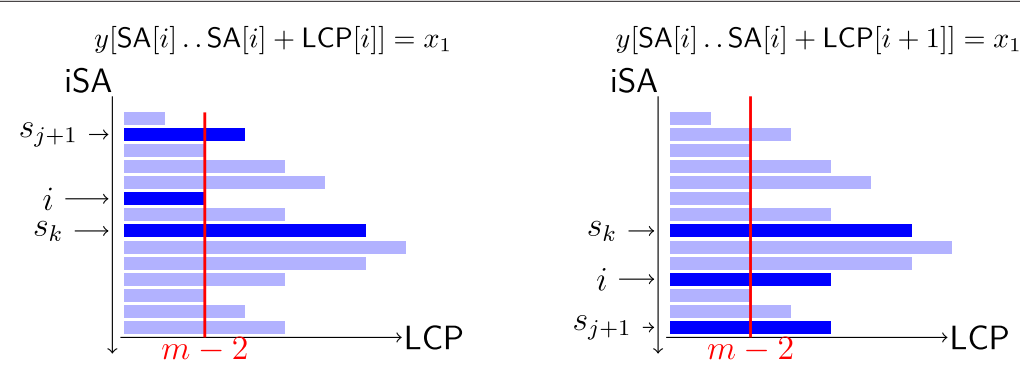

Figure 3 Illustration of Lemma 2. 
$n$ values, this step requires time and space $\mathcal{O}(n \sigma)$. For an example, see Figure 4.

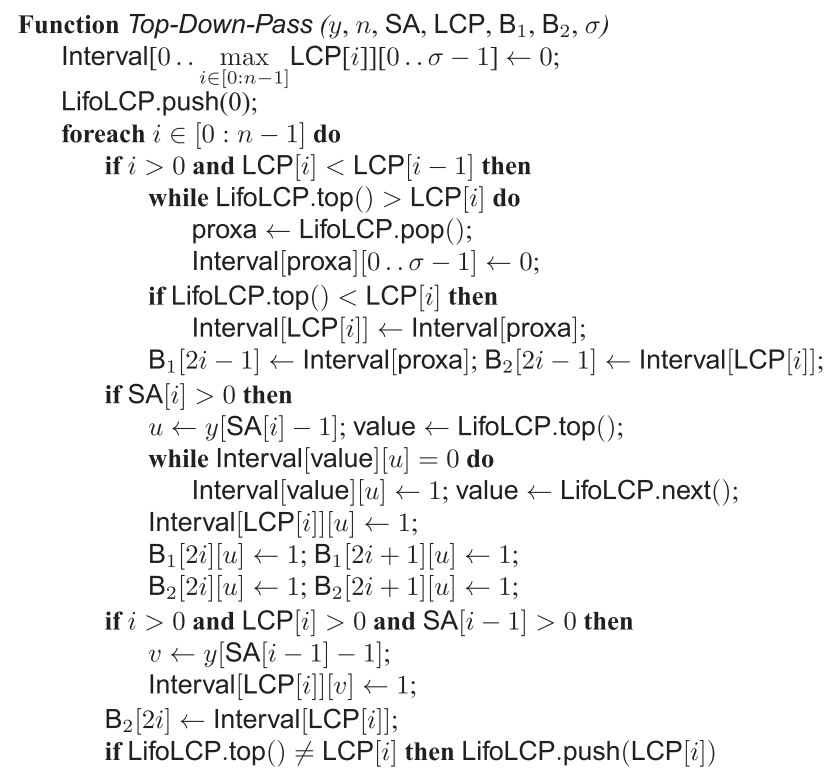

\section{Bottom-up pass}

Intuitively, the idea behind the bottom-up pass is the same as in the top-down pass except that in this instance, as we start from the bottom, the suffix $y[\mathrm{SA}[i] . . n-1]$ can share more than its prefix of length LCP $[i]$ with the previous suffixes in SA. Therefore we may need the elements of Interval that correspond to factors with an LCP value greater than $L C P[i]$ to correctly compute the arrays $\mathrm{B}_{1}$ and $B_{2}$. To achieve this, we maintain another stack LifoRem to copy the values from LifoLCP that are greater than LCP $[i]$. This extra stack allows us to keep in LifoLCP only values that are smaller or equal to $\mathrm{LCP}[i]$ without losing the additional information we require to correctly compute $B_{1}$ and $B_{2}$. At the end of the iteration, we will set to zero each element corresponding to a value in LifoRem and empty the stack. Thus to set an element of Interval to zero requires two operations more than in the first pass. As we consider at most $n$ values, this step requires time and space $\mathcal{O}(n \sigma)$.

Another difference between the top-down and bottomup passes is that in order to retain the information computed in the first pass, the second step is performed for each letter in $\mathrm{B}_{1}[2 i]$. As, for each LCP value read, we still add a letter only if is not already contained in the corresponding element of Interval, no more than $\sigma$ letters are added. Thus this step requires time and space $\mathcal{O}(n \sigma)$. For an example, see Figure 5.

Once we have computed arrays $B_{1}$ and $B_{2}$, we need to compare each element. If there is a difference, by Lemma 1, we can construct a minimal absent word. For an example, see Figure 6 . To ensure that we can report the minimal absent words in linear time, we must be able to report each one in constant time. To achieve this, we can represent them as a tuple $\langle a,(i, j)\rangle$, where for some word $x$ of length $m \geq 2$ that is a minimal absent word of $y$, the following holds: $x[0]=a$ and $x[1 . . m-1]=y[i . . j]$. Note that this representation uniquely identifies a minimal absent word and conversion from this encoding to the actual minimal absent word is trivial. Lemma 2 ensures us to be exhaustive. Therefore we obtain the following result.

Theorem 1. Algorithm MAW solves problem MINIMALABSENTWORDS in time and space $\mathcal{O}(n)$. (a)

\begin{tabular}{|c|c|c|}
\hline$j$ & $\mathrm{~B}_{1}$ & $\mathrm{~B}_{2}$ \\
\hline 0 & 00 & 00 \\
\hline 1 & 00 & 00 \\
\hline 2 & 10 & 10 \\
\hline 3 & 10 & 10 \\
\hline 4 & 01 & 11 \\
\hline 5 & 11 & 11 \\
\hline 6 & 01 & 11 \\
\hline 7 & 11 & 11 \\
\hline 8 & 01 & 11 \\
\hline 9 & 01 & 01 \\
\hline 10 & 10 & 11 \\
\hline 11 & 10 & 10 \\
\hline 12 & 10 & 10 \\
\hline 13 & 10 & 11 \\
\hline 14 & 10 & 11 \\
\hline
\end{tabular}

(b)

\begin{tabular}{|c|c|c|c|c|c|c|c|c|c|c|c|c|}
\hline$i$ & LCP & SA & \multicolumn{5}{|c|}{ Factor } & Interval $[0]$ & Interval[1] & Interval[2] & Interval[3] & Interval[4] \\
\hline \multirow[t]{2}{*}{0} & 0 & 0 & A & & & & & 00 & 00 & 00 & 00 & 00 \\
\hline & & & A & A & & & & & & & & \\
\hline \multirow[t]{2}{*}{1} & 1 & 1 & A & B & & & & 10 & 10 & 00 & 00 & 00 \\
\hline & & & A & B & A & B & A & & & & & \\
\hline \multirow[t]{2}{*}{2} & 4 & 3 & A & B & A & B & B & 11 & 11 & 00 & 00 & 11 \\
\hline & & & A & B & A & & & & & & & \\
\hline \multirow[t]{2}{*}{3} & 2 & 5 & A & B & B & & & 11 & 11 & 11 & 00 & 00 \\
\hline & & & A & & & & & & & & & \\
\hline \multirow[t]{2}{*}{4} & 0 & 7 & B & & & & & 11 & 00 & 00 & 00 & 00 \\
\hline & & & B & & & & & & & & & \\
\hline \multirow[t]{2}{*}{5} & 1 & 2 & B & A & & & & 11 & 11 & 00 & 00 & 00 \\
\hline & & & B & A & B & A & & & & & & \\
\hline \multirow[t]{2}{*}{6} & 3 & 4 & B & $\mathrm{A}$ & B & B & & 11 & 11 & 00 & 10 & 00 \\
\hline & & & B & $\mathrm{A}$ & & & & & & & & \\
\hline 7 & 1 & 6 & B & B & & & & 11 & 11 & 00 & 00 & 00 \\
\hline
\end{tabular}

Factors $S_{j}$ are in orange and violet; their longest proper prefixes are in orange only.

Figure 4 Top-down pass. (a) Arrays $B_{1}$ and $B_{2}$ obtained after the top-down pass for word $y=A A B A B A B B ;$ (b) Elements of array Interval at the end of each iteration of the top-down pass. 
(a)

\begin{tabular}{|c|c|c|}
\hline$j$ & $\mathrm{~B}_{1}$ & $\mathrm{~B}_{2}$ \\
\hline 0 & 11 & 11 \\
\hline 1 & 00 & 11 \\
\hline 2 & 11 & 11 \\
\hline 3 & 10 & 11 \\
\hline 4 & 01 & 11 \\
\hline 5 & 11 & 11 \\
\hline 6 & 01 & 11 \\
\hline 7 & 11 & 11 \\
\hline 8 & 11 & 11 \\
\hline 9 & 01 & 11 \\
\hline 10 & 10 & 11 \\
\hline 11 & 10 & 10 \\
\hline 12 & 10 & 10 \\
\hline 13 & 10 & 11 \\
\hline 14 & 10 & 11 \\
\hline
\end{tabular}

(b)

\begin{tabular}{|c|c|c|c|c|c|c|c|c|c|c|c|c|}
\hline$i$ & LCP & SA & \multicolumn{5}{|c|}{ Factor } & Interval $[0]$ & Interval[1] & Interval[2] & Interval[3] & Interval[4] \\
\hline \multirow[t]{2}{*}{7} & 1 & 6 & B & B & & & & 10 & 10 & 00 & 00 & 00 \\
\hline & & & B & A & & & & & & & & \\
\hline \multirow[t]{2}{*}{6} & 3 & 4 & B & A & B & B & & 10 & 10 & 00 & 10 & 00 \\
\hline & & & B & A & B & A & & & & & & \\
\hline \multirow[t]{2}{*}{5} & 1 & 2 & B & A & & & & 10 & 10 & 00 & 00 & 00 \\
\hline & & & B & & & & & & & & & \\
\hline \multirow[t]{2}{*}{4} & 0 & 7 & B & & & & & 11 & 00 & 00 & 00 & 00 \\
\hline & & & A & & & & & & & & & \\
\hline \multirow[t]{2}{*}{3} & 2 & 5 & A & B & B & & & 11 & 00 & 01 & 00 & 00 \\
\hline & & & A & B & A & & & & & & & \\
\hline \multirow[t]{2}{*}{2} & 4 & 3 & A & B & A & B & B & 11 & 00 & 01 & 00 & 01 \\
\hline & & & A & B & A & B & A & & & & & \\
\hline \multirow[t]{2}{*}{1} & 1 & 1 & A & B & & & & 11 & 11 & 00 & 00 & 00 \\
\hline & & & A & A & & & & & & & & \\
\hline 0 & 0 & 0 & A & & & & & 11 & 00 & 00 & 00 & 00 \\
\hline
\end{tabular}

Factors $S_{j}$ are in orange and violet; their longest proper prefixes are in orange only.

Figure 5 Bottom-up pass. (a) Arrays $B_{1}$ and $B_{2}$ obtained after the bottom-up pass for word $y=A A B A B A B B$; (b) Elements of array Interval at the end of each iteration of the bottom-up pass.

Function Bottom-Up-Pass ( $n$, SA, LCP, $\left.\mathrm{B}_{1}, \mathrm{~B}_{2}, \Sigma, \sigma\right)$

Interval $\left[0 \ldots \max _{i \in[0: n-1]} \operatorname{LCP}[i]\right][0 \ldots \sigma-1] \leftarrow 0$;

LifoLCP.push(0);

foreach $i \in[n-1: 0]$ do

proxa $\leftarrow \operatorname{LCP}[i]+1$; proxb $\leftarrow 1$;

if $i<n-1$ and $\operatorname{LCP}[i]<\operatorname{LCP}[i+1]$ then

while LifoLCP.top ()$>\operatorname{LCP}[i]$ do

proxa $\leftarrow$ LifoLCP.pop () ;

LifoRem.push(proxa);

if $\operatorname{LifoLCP.top}()<\operatorname{LCP}[i]$ then Interval $[\mathrm{LCP}[i]] \leftarrow$ Interval $[$ proxa]

foreach $k \in \Sigma: \mathrm{B}_{1}[2 i][k]=1$ do

value $\leftarrow$ LifoLCP.top () ;

while Interval $[$ value $][k]=0$ do

Interval $[$ value $][k] \leftarrow 1$; value $\leftarrow$ LifoLCP.next()

Interval $[\mathrm{LCP}[i]][k] \leftarrow 1$;

$\mathrm{B}_{2}[2 i] \leftarrow \mathrm{B}_{2}[2 i] \quad$ bit-or Interval $[\mathrm{LCP}[i]]$

$\mathrm{B}_{2}[2 i+1] \leftarrow \mathrm{B}_{2}[2 i+1]$ bit-or Interval $[\mathrm{LCP}[i+1]]$;

$\mathrm{B}_{1}[2 i+1] \leftarrow \mathrm{B}_{1}[2 i+1]$ bit-or Interval[proxb];

proxb $\leftarrow$ proxa;

$\mathrm{B}_{1}[2 i] \leftarrow \mathrm{B}_{1}[2 i]$ bit-or Interval $[$ proxa $]$;

while LifoRem not empty do

value $\leftarrow$ LifoRem.pop () ; Interval[value $][0 \ldots \sigma-1] \leftarrow 0$;

if LifoLCP.top ()$\neq \operatorname{LCP}[i]$ then LifoLCP.push $(\operatorname{LCP}[i])$

\section{Results and discussion}

The experiments were conducted on a Desktop PC using one core of Intel Xeon E5540 CPU at $2.5 \mathrm{GHz}$ and 32GB of main memory under 64-bit GNU/Linux. All programs were compiled with gcc version 4.6.3 at optimisation level 3 (-O3). Time and memory measurements were taken using the GNU/Linux time command.

\section{Implementation}

We implemented algorithm MAW as a program to compute all minimal absent words of a given sequence. The program was implemented in the $C$ programming language and developed under GNU/Linux operating system. It takes as input arguments a file in (Multi)FASTA format and the minimal and maximal length of minimal absent words to be outputted; and then produces a file with all minimal absent words of length within this range as output. The implementation is distributed under the GNU General Public License (GPL), and it is

\begin{tabular}{|c|c|c|c|c|c|c|c|c|c|}
\hline$j$ & $\mathrm{~B}_{1}$ & $\mathrm{~B}_{2}$ & \multicolumn{5}{|c|}{ Factor } & Minimal absent words & Tuple representation \\
\hline 0 & 11 & 11 & A & & & & & & \\
\hline 1 & 00 & 11 & A & A & & & & AAA, BAA & $<\mathrm{A},(0,1)>,<\mathrm{B},(0,1)>$ \\
\hline 2 & 11 & 11 & A & B & & & & & \\
\hline 3 & 10 & 11 & A & B & A & B & A & BABABA & $<\mathrm{B},(1,5)>$ \\
\hline 4 & 01 & 11 & A & B & A & B & B & AABABB & $<\mathrm{A},(3,7)>$ \\
\hline 5 & 11 & 11 & A & B & A & & & & \\
\hline 6 & 01 & 11 & A & B & B & & & AABB & $<\mathrm{A},(5,7)>$ \\
\hline 7 & 11 & 11 & A & & & & & & \\
\hline 8 & 11 & 11 & B & & & & & & \\
\hline 9 & 01 & 11 & B & & & de & no & consider this row as it & corresponds to the end of the word $y$ \\
\hline 10 & 10 & 11 & B & A & & & & BBA & $<\mathrm{B},(2,3)>$ \\
\hline 11 & 10 & 10 & B & A & B & A & & & \\
\hline 12 & 10 & 10 & B & A & B & B & & & \\
\hline 13 & 10 & 11 & B & A & & & & BBA & This is a duplicate so we ignore it \\
\hline 14 & 10 & 11 & B & B & & & & BBB & $<\mathrm{B},(6,7)>$ \\
\hline
\end{tabular}

Figure 6 Minimal absent words of word $y=A A B A B A B B$; we find seven minimal absent words $\{A A A, A A B A B B, A A B B, B A A, B A B A B A$, $\mathrm{BBA}, \mathrm{BBB}\}$. 
available at http://github.com/solonas13/maw, which is set up for maintaining the source code and the man-page documentation.

\section{Datasets}

We considered the genomes of thirteen bacteria and four case-study eukaryotes (Table 1), all obtained from the NCBI database (ftp://ftp.ncbi.nih.gov/genomes/).

\section{Correctness}

To test the correctness of our implementation, we compared it against the implementation of Pinho et al. [6], which we denote here by PFG. In particular, we counted the number of minimal absent words, for lengths 11,14 , 17 , and 24, in the genomes of the thirteen bacteria listed in Table 1 . We considered only the $5^{\prime} \rightarrow 3^{\prime}$ DNA strand.

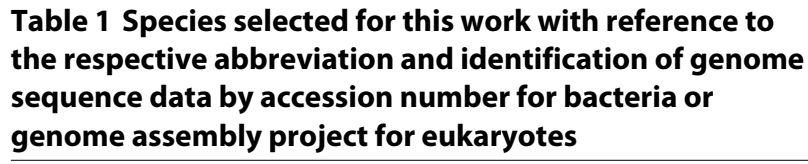

\begin{tabular}{|c|c|c|}
\hline Species & Abbreviation & Genome reference \\
\hline \multicolumn{3}{|l|}{ Bacteria } \\
\hline $\begin{array}{l}\text { Bacillus anthracis } \\
\text { strain Ames }\end{array}$ & $\mathrm{Ba}$ & NC003997 \\
\hline $\begin{array}{l}\text { Bacillus subtilis } \\
\text { strain } 168\end{array}$ & Bs & NC000964 \\
\hline $\begin{array}{l}\text { Escherichia coli strain K-12 } \\
\text { substrain MG1655 }\end{array}$ & Ec & NC000913 \\
\hline $\begin{array}{l}\text { Haemophilus influenzae } \\
\text { strain Rd KW20 }\end{array}$ & $\mathrm{Hi}$ & NC000907 \\
\hline $\begin{array}{l}\text { Helicobacter pylori } \\
\text { strain } 26695\end{array}$ & $\mathrm{Hp}$ & NC000915 \\
\hline $\begin{array}{l}\text { Lactobacillus casei } \\
\text { strain BL23 }\end{array}$ & LC & NC010999 \\
\hline $\begin{array}{l}\text { Lactococcus lactis } \\
\text { strain } / 11403\end{array}$ & $\mathrm{LI}$ & NC002662 \\
\hline $\begin{array}{l}\text { Mycoplasma genitalium } \\
\text { strain G37 }\end{array}$ & $\mathrm{Mg}$ & NC000908 \\
\hline $\begin{array}{l}\text { Staphylococcus aureus } \\
\text { strain N315 }\end{array}$ & Sa & NC002745 \\
\hline $\begin{array}{l}\text { Streptococcus pneumoniae } \\
\text { strain CGSP14 }\end{array}$ & $\mathrm{Sp}$ & NC010582 \\
\hline $\begin{array}{l}\text { Xanthomonas campestris } \\
\text { strain } 8004\end{array}$ & $X_{c}$ & NC007086 \\
\hline \multicolumn{3}{|l|}{ Eukaryotes } \\
\hline $\begin{array}{l}\text { Arabidopsis thaliana } \\
\text { (thale cress) }\end{array}$ & At & AGl release 7.2 \\
\hline $\begin{array}{l}\text { Drosophila melanogaster } \\
\text { (fruit fly) }\end{array}$ & $\mathrm{Dm}$ & FlyBase release 5 \\
\hline Homo sapiens (human) & $\mathrm{Hs}$ & build 38 \\
\hline Mus musculus (mouse) & $\mathrm{Mm}$ & build 38 \\
\hline
\end{tabular}

Table 2 Number of minimal absent words of lengths 11 , 14,17 , and 24 in the genomes of thirteen bacteria

\begin{tabular}{llllll}
\hline Species & Genome size (bp) & $\mathbf{M}_{\mathbf{1 1}}$ & $\mathbf{M}_{\mathbf{1 4}}$ & $\mathbf{M}_{\mathbf{1 7}}$ & $\mathbf{M}_{\mathbf{2 4}}$ \\
\hline $\mathrm{Ba}$ & $5,227,293$ & $1,113,398$ & $1,001,357$ & 32,432 & 46 \\
$\mathrm{Bs}$ & $4,214,630$ & 951,273 & $1,703,309$ & 86,372 & 226 \\
EC & $4,639,675$ & $1,072,074$ & $1,125,653$ & 36,395 & 247 \\
$\mathrm{Hi}$ & $1,830,023$ & 722,860 & 294,353 & 12,158 & 91 \\
$\mathrm{Hp}$ & $1,667,825$ & 564,308 & 336,122 & 19,276 & 75 \\
LC & $3,079,196$ & $1,126,363$ & 502,861 & 13,083 & 246 \\
LI & $2,365,589$ & 764,006 & 507,490 & 25,667 & 183 \\
Mg & $1,664,957$ & 246,342 & 66,324 & 2,737 & 28 \\
Sa & $2,814,816$ & 755,483 & 704,147 & 32,054 & 138 \\
Sp & $2,209,198$ & 904,815 & 327,713 & 10,390 & 234 \\
Xc & $5,148,708$ & 804,034 & $1,746,214$ & 179,346 & 633 \\
\hline
\end{tabular}

Table 2 depicts the number of minimal absent words in these sequences. We denote by $M_{11}, M_{14}, M_{17}$, and $M_{24}$ the size of the resulting sets of minimal absent words for lengths $11,14,17$, and 24 respectively. Identical number of minimal absent words for these lengths were also reported by PFG, suggesting that our implementation is correct.

\section{Efficiency}

To evaluate the efficiency of our implementation, we compared it against the corresponding performance of PFG, which is currently the fastest available implementation for computing minimal absent words. Notice that this evaluation depends heavily on the suffix array con-

Table 3 Elapsed-time comparison of MAW and PFG for computing all minimal absent words in the genome of Arabidopsis thaliana and Drosophila melanogaster

\begin{tabular}{llll}
\hline \multicolumn{4}{c}{ (a) At } \\
\hline Chromosome & Size (bp) & MAW (s) & PFG (s) \\
\hline 1 & $30,427,671$ & 40.20 & 51.90 \\
2 & $19,698,289$ & 25.86 & 32.94 \\
3 & $23,459,830$ & 30.84 & 42.30 \\
4 & $18,585,056$ & 24.65 & 31.42 \\
5 & $26,975,502$ & 35.38 & 48.91 \\
\hline & (b) Dm & & PFG (s) \\
\hline Chromosome & Size (bp) & MAW (s) & 40.85 \\
\hline $2 \mathrm{~L}$ & $23,011,544$ & 30.01 & 38.38 \\
$2 \mathrm{R}$ & $21,146,708$ & 27.52 & 45.13 \\
$3 \mathrm{~L}$ & $24,543,557$ & 32.00 & 48.36 \\
$3 \mathrm{R}$ & $27,905,053$ & 36.44 & 40.09 \\
$X$ & $22,422,827$ & 29.38 & \\
\hline
\end{tabular}



Table 4 Elapsed-time comparison of MAW and PFG for
computing all minimal absent words in the genome of
Homo Sapiens and Mus musculus

\begin{tabular}{|c|c|c|c|}
\hline \multicolumn{4}{|c|}{ (a) $\mathrm{Hs}$} \\
\hline Chromosome & Size (bp) & MAW (s) & PFG (s) \\
\hline 1 & $248,956,422$ & 426.39 & 972.52 \\
\hline 2 & $242,193,529$ & 423.19 & 772.89 \\
\hline 3 & $198,295,559$ & 353.60 & 645.45 \\
\hline 4 & $190,214,555$ & 339.02 & 616.26 \\
\hline 5 & $181,538,259$ & 342.53 & 577.05 \\
\hline 6 & $170,805,979$ & 299.72 & 538.34 \\
\hline 7 & $159,345,973$ & 305.26 & 491.32 \\
\hline 8 & $145,138,636$ & 254.17 & 437.18 \\
\hline 9 & $138,394,717$ & 235.14 & 356.08 \\
\hline 10 & $133,797,422$ & 235.38 & 392.45 \\
\hline 11 & $135,086,622$ & 236.80 & 379.15 \\
\hline 12 & $133,275,309$ & 235.14 & 390.46 \\
\hline 13 & $114,364,328$ & 191.64 & 269.52 \\
\hline 14 & $107,043,718$ & 178.00 & 240.93 \\
\hline 15 & $101,991,189$ & 167.89 & 222.98 \\
\hline 16 & $90,338,345$ & 153.07 & 198.49 \\
\hline 17 & $83,257,441$ & 144.32 & 207.02 \\
\hline 18 & $80,373,285$ & 137.68 & 199.44 \\
\hline 19 & $58,617,616$ & 100.95 & 126.82 \\
\hline 20 & $64,444,167$ & 109.80 & 144.83 \\
\hline 21 & $46,709,983$ & 74.60 & 74.65 \\
\hline 22 & $50,818,468$ & 70.49 & 73.34 \\
\hline$x$ & $156,040,895$ & 275.14 & 457.2 \\
\hline Y & $57,227,415$ & 60.85 & 62.34 \\
\hline
\end{tabular}

\begin{tabular}{llll}
\hline Chromosome & Size (bp) & MAW (s) & PFG (s) \\
\hline 1 & $197,195,432$ & 340.59 & 599.86 \\
2 & $181,748,087$ & 316.17 & 578.2 \\
3 & $159,599,783$ & 274.46 & 506.73 \\
4 & $155,630,120$ & 266.67 & 473.97 \\
5 & $152,537,259$ & 260.50 & 424.24 \\
6 & $149,517,037$ & 256.36 & 455.11 \\
7 & $152,524,553$ & 257.65 & 413.37 \\
8 & $131,738,871$ & 223.09 & 344.92 \\
9 & $124,076,172$ & 210.37 & 334.25 \\
10 & $129,993,255$ & 222.36 & 363.34 \\
11 & $121,843,856$ & 208.55 & 324.54 \\
12 & $121,257,530$ & 205.09 & 324.79 \\
13 & $120,284,312$ & 204.80 & 314.56 \\
14 & $125,194,864$ & 212.59 & 336.49 \\
15 & $103,494,974$ & 175.21 & 265.92 \\
16 & $98,319,150$ & 166.10 & 249.03
\end{tabular}

Table 4 Elapsed-time comparison of MAW and PFG for computing all minimal absent words in the genome of Homo Sapiens and Mus musculus (Continued)

\begin{tabular}{llll}
\hline 17 & $95,272,651$ & 160.70 & 232.79 \\
18 & $90,772,031$ & 153.40 & 223.56 \\
19 & $61,342,430$ & 101.89 & 125.85 \\
$X$ & $166,650,296$ & 282.21 & 503.98 \\
$Y$ & $91,744,698$ & 141.79 & 251 \\
\hline
\end{tabular}

struction implementation used; and that PFG uses a less optimised implementation for this construction than the one used by MAW. We computed all minimal absent words for each chromosome sequence of the genomes of the four eukaryotes listed in Table 1 . We considered both the $5^{\prime} \rightarrow 3^{\prime}$ and the $3^{\prime} \rightarrow 5^{\prime}$ DNA strands. Tables 3 and 4 depict elapsed-time comparisons of MAW and PFG. We observe that PFG scales mostly linearly. MAW also scales linearly and is the fastest in all cases. It accelerates the computations by more than a factor of 2 , when the sequence length grows, compared to PFG. Figure 7 corresponds to the measurements in Table 4: it plots chromosome sequence length versus elapsed time for computing all minimal absent words in the genomes of Homo Sapiens and Mus musculus using MAW and PFG. MAW also reduces the memory requirements by a factor of 5 compared to PFG. The maximum allocated memory (per task) was 6GB for MAW and 30GB for PFG.

To further evaluate the efficiency of our implementation, we compared it against the corresponding performance of PFG using synthetic data. As basic dataset we used chromosome 1 of Hs. We created five instances $\mathrm{S}_{1}, \mathrm{~S}_{2}, \mathrm{~S}_{3}, \mathrm{~S}_{4}$, and $\mathrm{S}_{5}$ of this sequence by randomly choosing $10 \%, 20 \%, 30 \%, 40 \%$, and $50 \%$ of the positions, respectively, and randomly replacing the corresponding letters to one of the four letters of the DNA alphabet. We computed all minimal absent words for each instance. We considered both the $5^{\prime} \rightarrow 3^{\prime}$ and the $3^{\prime} \rightarrow 5^{\prime}$ DNA strands. Table 5 depicts elapsed-time comparisons of MAW and PFG. MAW is the fastest in all cases.

\section{Conclusions}

In this article, we presented the first $\mathcal{O}(n)$-time and $\mathcal{O}(n)$ space algorithm for computing all minimal absent words based on the construction of suffix arrays. In addition, we provided the respective implementation of this algorithm. Experimental results show that this implementation outperforms existing tools, both in terms of speed and memory.

In a typical application, one would be interested in computing minimal absent words in the whole genome 


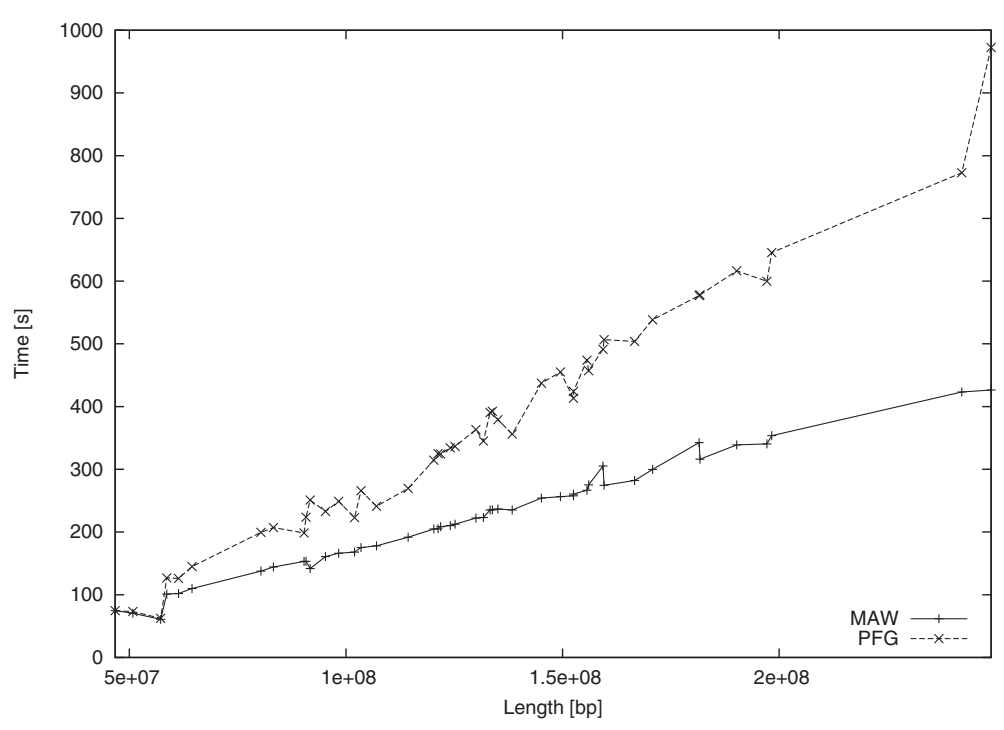

Figure 7 Chromosome sequence length versus elapsed time for computing all minimal absent words in the genomes of Homo Sapiens and Mus musculus using MAW and PFG.

for a set of species under study $[11,12]$. Hence, we consider the improvements described in this article to be of great importance. Our immediate target is twofold: first, explore the possibility of implementing the presented algorithm for symmetric multiprocessing systems; and second, devise and implement a fast space-efficient solution for this problem based on the construction of compressed full-text indexes.

\section{Availability and requirements}

- Project name: MAW

- Project home page: http://github.com/solonas13/ maw

- Operating system: GNU/Linux

- Programming language: $\mathrm{C}$

- Other requirements: compiler gcc version 4.6 .3 or higher

- License: GNU GPL

- Any restrictions to use by non-academics: licence needed

Table 5 Elapsed-time comparison of MAW and PFG for computing all minimal absent words in synthetic data

\begin{tabular}{llll}
\hline Sequence & Size (bp) & MAW (s) & PFG (s) \\
\hline$S_{1}$ & $248,956,422$ & 435.63 & 746.93 \\
$S_{2}$ & $248,956,422$ & 438.52 & 733.69 \\
$S_{3}$ & $248,956,422$ & 444.62 & 726.34 \\
$S_{4}$ & $248,956,422$ & 444.06 & 743.29 \\
$S_{5}$ & $248,956,422$ & 449.25 & 741.01 \\
\hline
\end{tabular}

\section{Competing interests}

The authors declare that they have no competing interests.

\section{Authors' contributions}

SPP designed the study. AH and CB devised the algorithms. AH and SPP implemented the algorithms. SPP conducted the experiments. $A H, C B$, and $L M$ wrote the manuscript. The final version of the manuscript is approved by all authors.

\section{Acknowledgements}

The publication costs for this article were funded by the Open Access funding scheme of King's College London. CB is supported by an EPSRC grant (Doctoral Training Grant \#EP/J500252/1). SPP is supported by a Research Grant (\#RG130720) awarded by the Royal Society. The authors would like to thank the CRIHAN (Centre des Ressources Informatiques de HAute-Normandie, France) for the excellent support and the available computation resources that have been used during the development and test phases. The authors would like to thank Maxime Crochemore for introducing this problem and valuable discussions.

\section{Author details}

${ }^{1}$ Department of Informatics, King's College London, The Strand, WC2R 2LS London, UK. ${ }^{2}$ Inria Saclay-Île de France, AMIB, Bâtiment Alan Turing, Palaiseau, France. ${ }^{3}$ Laboratoire d'Informatique de l'École Polytechnique (LIX), CNRS UMR 7161, Palaiseau, France. ${ }^{4}$ University of Rouen, LITIS EA 4108, TIBS, Rouen, France.

Received: 27 June 2014 Accepted: 18 November 2014

Published online: 20 December 2014

\section{References}

1. Haubold B, Pierstorff N, Möller F, Wiehe T: Genome comparison without alignment using shortest unique substrings. BMC Bioinformatics 2005 , 6:123.

2. Domazet-Lošo M, Haubold B: Efficient estimation of pairwise distances between genomes. Bioinformatics 2009, 25(24):3221-3227.

3. Hampikian $G$, Andersen $T$ : Absent sequences: nullomers and primes. In Pacific Symposium on Biocomputing. Edited by Altman RB, Dunker AK, Hunter L, Murray T, Klein TE. Maui, Hawaii, USA: World Scientific; 2007:355-366.

4. Herold J, Kurtz S, Giegerich R: Efficient computation of absent words in genomic sequences. BMC Bioinformatics 2008, 9:1-9. 
5. Wu ZD, Jiang T, Su WJ: Efficient computation of shortest absent words in a genomic sequence. Inform Process Lett 2010, 110(14-15):596-601.

6. Pinho AJ, Ferreira PJSG, Garcia SP: On finding minimal absent words. BMC Bioinformatics 2009 11. [http://dx.doi.org/10.1186/1471-2105-10137]

7. Crochemore M, Mignosi F, Restivo A: Automata and forbidden words. Inform Process Lett 1998, 67:111-117.

8. Mignosi F, Restivo A, Sciortino M: Words and forbidden factors. Theor Comput Sci 2002, 273(1-2):99-117.

9. Fici G: Minimal forbidden words and applications. PhD thesis. Université de Marne-la-Vallée; 2006.

10. Acquisti C, Poste G, Curtiss D, Kumar S: Nullomers: really a matter of natural selection? PLOS ONE 2007, 2(10):e1022.

11. Garcia SP, Pinho AJ, Rodrigues JM, Bastos CA, Ferreira PJ: Minimal absent words in prokaryotic and eukaryotic genomes. PLOS ONE 2011, 6(1):e16065.

12. Garcia SP, Pinho AJ: Minimal absent words in four human genome assemblies. PLOS ONE 2011, 6(12):e29344.

13. Chairungsee $\mathrm{S}$, Crochemore $\mathrm{M}$ : Using minimal absent words to build phylogeny. Theor Comput Sci 2012, 450(0):109-116.

14. Belazzougui D, Cunial F, Kärkkäinen J, Mäkinen V: Versatile succinct representations of the bidirectional burrows-wheeler transform. In ESA, Volume 8125 of Lecture Notes in Computer Science. Edited by Bodlaender HL, Italiano GF. Sophia Antipolis, France: Springer; 2013:133-144.

15. Manber U, Myers EW: Suffix arrays: a new method for on-line string searches. SIAM J Comput 1993, 22(5):935-948.

16. Nong G, Zhang S, Chan WH: Linear suffix array construction by almost pure induced-sorting. In Proceedings of the 2009 Data Compression Conference, DCC '09. Washington, DC, USA: IEEE Computer Society; 2009:193-202

17. Fischer J: Inducing the LCP-Array. In Algorithms and Data Structures 12th International Symposium, Volume 6844 of Lecture Notes in Computer Science. Edited by Dehne F, lacono J, Sack JR. New York, NY, USA: Springer; 2011:374-385

Submit your next manuscript to BioMed Central and take full advantage of:

- Convenient online submission

- Thorough peer review

- No space constraints or color figure charges

- Immediate publication on acceptance

- Inclusion in PubMed, CAS, Scopus and Google Scholar

- Research which is freely available for redistribution

Submit your manuscript at www.biomedcentral.com/submit
C Biomed Central 